\title{
Powers of Edge Ideals with Linear Resolutions
}

\author{
Nursel Erey*
}

\begin{abstract}
We show that if $G$ is a gap-free and diamond-free graph, then $I(G)^{s}$ has a linear minimal free resolution for every $s \geq 2$.
\end{abstract}

\section{Introduction}

Let $S=k\left[x_{1}, \ldots, x_{n}\right]$ be a polynomial ring and let $G$ be a finite simple graph with edge ideal $I(G)$. There has been recent interest in studying the asymptotic regularity of powers of edge ideals for various families of graphs [2, 3, 8, 10, 11]. It is known [9] that when $I$ is a graded ideal, regularity of powers $I^{s}$ is a linear function for large $s$. In particular, for any graph $G$, there exist constants $c$ and $s_{0}$ such that $\operatorname{reg}\left(I(G)^{s}\right)=2 s+c$ for all $s \geq s_{0}$. A special case of interest is to combinatorially characterize when the powers of edge ideals have eventually linear minimal free resolutions. Since the sth power of the edge ideal is generated in degree $2 s$, the ideal $I(G)^{s}$ has a linear minimal free resolution if and only if $c=0$ in the given formula.

It was proved by Francisco, Hà and Van Tuyl that if some power of $I(G)$ has linear minimal free resolution, then the complement graph $G^{c}$ has no induced 4-cycles, i.e., $G$ is gap-free. In this direction, Nevo and Peeva [1] raised the following question.

Question 1.1. Is it true that a graph $G$ is gap-free if and only if $I(G)^{s}$ has a linear minimal free resolution for every $s \gg 0$ ?

This question is known to have a positive answer when the regularity of the edge ideal is 2. In fact, Herzog, Hibi and Zheng [8] proved that if $I(G)$ has a linear minimal free resolution, then so does $I(G)^{s}$ for all $s \geq 1$. Banerjee [2] proved that if $G$ is a gap-free and cricket-free graph, then $I(G)^{s}$ has linear minimal free resolution for every $s \geq 2$. In this paper, we provide a new family of gap-free graphs for which the second and higher powers of edge ideals have linear minimal free resolutions.

\footnotetext{
${ }^{*}$ Department of Mathematics, North Dakota State University, Fargo, ND e-mail: nurselerey@gmail.com 2010 Mathematics Subject Classification. 13D02, 05E40 Keywords: Edge Ideal, Castelnuovo-Mumford Regularity
} 


\section{Preliminaries}

\subsection{Graph theory}

Throughout this paper we consider finite simple graphs, i.e., graphs with no loops or multiple edges. For any graph $G$ we write $V(G)$ and $E(G)$ respectively for the vertex and edge sets of the graph. We say two vertices $u$ and $v$ are adjacent and write $u v \in G$, if there is an edge between them. The set $N(u)=\{v: v$ is adjacent to $u\}$ is called the neighbor set of $u$. Any $v \in N(u)$ is called a neighbor of $u$. A vertex is called an isolated vertex if it has no neighbors. A complete graph (or clique) is a graph such that every pair of vertices are adjacent. A complete graph on $n$ vertices is denoted by $K_{n}$.

A graph $H$ is called a subgraph of $G$ if the vertex and edge sets of $H$ are contained respectively in those of $G$. A subgraph $H$ of $G$ is called an induced subgraph if $u v \in G$ implies $u v \in H$ for every $u, v \in V(H)$. A subset $A$ of the vertices of $G$ is called independent if $u v \notin G$ for every $u, v \in A$. A dominating clique of a graph $G$ is a complete subgraph $H$ of $G$ such that every vertex of $G$ either belongs to $H$ or is adjacent to some vertex of $H$.

For any graph $K$, we say $G$ is $K$-free if $K$ is not an induced subgraph of $G$. The clique number of $G$, denoted $\omega(G)$, is the number of vertices in a maximum clique of $G$. For any vertex $v$ of $G$, the graph $G-v$ denotes the induced subgraph of $G$ which is obtained from $G$ by removing the vertex $v$. The complement of a graph $G$, denoted $G^{c}$, is a graph on the same vertex set such that for every vertex $u$ and $v, u v \in G^{c}$ if $u v \notin G$.

Let $F$ and $G$ be graphs with no common vertices and let $v \in V(G)$. We say $G^{\prime}$ is obtained from $G$ by substituting $F$ for $v$ if the vertex $v$ is replaced by $F$ and all the vertices of $N(v)$ are adjacent to all the vertices of $F$, i.e., $V\left(G^{\prime}\right)=(V(G) \cup V(F)) \backslash\{v\}$ and $E\left(G^{\prime}\right)=E(G-v) \cup E(F) \cup\{a b: a \in N(v), b \in V(F)\}$. For a vertex $v \in V(G)$ and a positive integer $k$, we say $G^{\prime}$ is obtained from $G$ by multiplying $v$ by $k$ when $G^{\prime}$ is formed by substituting an independent set $A$ of $k$ vertices for $v$.

A cycle graph with vertices $v_{1}, \ldots, v_{n}$ and edges $v_{1} v_{2}, \ldots, v_{n-1} v_{n}, v_{n} v_{1}$ is denoted by $C_{n}=\left(v_{1} v_{2} \ldots v_{n}\right)$. The complement of a cycle graph is called an anticycle. A path in a graph $G$ is a sequence of vertices $u_{1}, \ldots, u_{n}$ such that $u_{i} u_{i+1} \in G$ for all $1 \leq i \leq n-1$. A path graph on $n$ vertices, denoted by $P_{n}$, is a graph with $n$ distinct vertices $u_{1}, \ldots, u_{n}$ and $n-1$ edges $u_{1} u_{2}, u_{2} u_{3}, \ldots, u_{n-1} u_{n}$. A graph which is isomorphic to the graph with vertices $a, b, c, d$ and edges $a b, b c, a c, a d, c d$ is called a diamond. A graph which is isomorphic to the graph with vertices $w_{1}, w_{2}, w_{3}, w_{4}, w_{5}$ and edges $w_{1} w_{3}, w_{2} w_{3}, w_{3} w_{4}, w_{3} w_{5}, w_{4} w_{5}$ is called a cricket.

A graph $G$ is called chordal if every induced cycle in $G$ has at most three vertices. A bipartite graph is a graph that does not contain any odd-length cycles. Two disjoint edges $u v$ and $a b$ of $G$ are said to form a gap if the induced subgraph of $G$ on the vertices $u, v, a, b$ has only two edges. A graph $G$ is called gap-free if no two edges form a gap, or equivalently, $G^{c}$ is $C_{4}$-free.

For any $v \in V(G)$ and any induced subgraph $H$ of $G$, the distance between $v$ and $H$ is the number of edges in a shortest path from $v$ to a vertex of $H$. In particular, the distance between $v$ and $H$ is zero when $v \in H$. 


\subsection{Castelnuovo-Mumford regularity of monomial ideals}

Let $S=k\left[x_{1}, \ldots, x_{n}\right]$ be a polynomial ring over a field $k$. The Castelnuovo-Mumford regularity of a monomial ideal $I$ is given by

$$
\operatorname{reg}(I)=\max \left\{j-i: b_{i, j}(I) \neq 0\right\}
$$

where $b_{i, j}(I)$ are the graded Betti numbers of $I$. If $G$ is a graph on the vertices $x_{1}, \ldots, x_{n}$, then the edge ideal of $G$ is defined as

$$
I(G)=(x y: x y \text { is an edge of } G) .
$$

The method of polarization reduces the study of minimal free resolutions of monomial ideals to that of square-free monomial ideals. Therefore quadratic monomial ideals can be studied via edge ideals. We recall the following well-known results.

Theorem 2.1. [7, Corollary 1.6.3] Let $I \subseteq S$ be a monomial ideal and let $I^{\text {pol }}$ be its polarization. Then $\operatorname{reg}(I)=\operatorname{reg}\left(I^{\mathrm{pol}}\right)$.

Lemma 2.2. If $I \subseteq S$ is a monomial ideal, then $\operatorname{reg}(I, x) \leq \operatorname{reg}(I)$ for any variable $x$.

We will make use of the following theorem to bound the regularity of monomial ideals.

Theorem 2.3. [5, Lemma 2.10][2, Lemma 2.11] Let $I \subseteq S$ be a monomial ideal, and let $m$ be a monomial of degree $d$. Then

$$
\operatorname{reg}(I) \leq \max \{\operatorname{reg}(I: m)+d, \operatorname{reg}(I, m)\} .
$$

Moreover, if $m$ is a variable $x$ appearing in $I$, then $\operatorname{reg}(I)$ is equal to one of these terms.

The next result is a restatement of Theorem 2.3 in terms of edge ideals. Note that for any vertex $x$ of $G$, the set st $x$ stands for $N(x) \cup\{x\}$.

Theorem 2.4. [5, Lemma 3.1] Let $x$ be a vertex of $G$ with neighbors $y_{1}, \ldots, y_{m}$. Then

$$
\operatorname{reg}(I(G)) \leq \max \{\operatorname{reg}(I(G-\operatorname{st} x))+1, \operatorname{reg}(I(G-x))\} .
$$

Moreover, $\operatorname{reg}(I(G))$ is equal to one of these terms.

Banerjee [2] introduced the following total order on the minimal monomial generators of powers of edge ideals.

Definition 2.5. [2, Discussion 4.1] Let I be an edge ideal which is minimally generated by the monomials $L_{1}, \ldots, L_{k}$. Consider the order $L_{1}>L_{2}>\cdots>L_{k}$ and let $n \geq 2$. For any minimal monomial generator $M$ of $I^{n}$, the expression $M=L_{1}^{a_{1}} L_{2}^{a_{2}} \ldots L_{k}^{a_{k}}$ is called maximal if for all expressions $M=L_{1}^{b_{2}} \ldots L_{k}^{b_{k}}$, we have $\left(a_{1}, \ldots, a_{k}\right)>_{\operatorname{lex}}\left(b_{1}, \ldots, b_{k}\right)$. Given two minimal monomial generators $M, N$ of $I^{n}$ with maximal expressions $M=L_{1}^{a_{1}} L_{2}^{a_{2}} \ldots L_{k}^{a_{k}}$ and $N=L_{1}^{c_{1}} L_{2}^{c_{2}} \ldots L_{k}^{c_{k}}$ we set $M>N$ if $\left(a_{1}, \ldots, a_{k}\right)>_{\operatorname{lex}}\left(c_{1}, \ldots, c_{k}\right)$. We let $L^{(n)}$ denote the totally ordered set of minimal monomial generators of $I^{n}$. 
The following theorem follows from [2, Theorem 4.12].

Theorem 2.6. [2] Let $I=\left(L_{1}, \ldots, L_{k}\right)$ be an edge ideal and let $s \geq 1$. Let $L^{(s)}: L_{1}^{(s)}>$ $L_{2}^{(s)}>\cdots>L_{r}^{(s)}$ be the order on the minimal monomial generators of $I^{s}$ induced by the order $L_{1}>\cdots>L_{k}$ as described in Definition 2.5. Then for every $1 \leq \ell \leq r-1$,

$$
\left(\left(I^{s+1}, L_{1}^{(s)}, \ldots, L_{\ell}^{(s)}\right): L_{\ell+1}^{(s)}\right)=\left(\left(I^{s+1}: L_{\ell+1}^{(s)}\right), \text { some variables }\right) .
$$

As a consequence of the theorem above, the following result was obtained in [2], which gives a sufficient condition for powers of edge ideals to have linear resolutions.

Theorem 2.7. [2, Corollary 5.3] Let $G$ be a graph with edge ideal $I=I(G)$. If $\operatorname{reg}(I) \leq 4$ and for all $s \geq 1$ and for each minimal monomial generator $m$ of $I^{s}, \operatorname{reg}\left(I^{s+1}: m\right) \leq 2$, then $I^{t}$ has linear minimal free resolution for every $t \geq 2$.

The following classical result of Fröberg [6] gives a combinatorial characterization of edge ideals which have linear minimal free resolutions.

Theorem 2.8. [6, Theorem 1] The minimal free resolution of $I(G)$ is linear if and only if the complement graph $G^{c}$ is chordal.

\subsection{Even-connection and gap-free graphs}

In [2, Definition 6.2] the concept of even-connectedness was defined in order to describe in combinatorial terms the minimal generators of certain colon ideals of powers of edge ideals.

Definition 2.9. Let $G$ be a graph. Two vertices $u$ and $v$ in $G$ are said to be evenconnected with respect to an s-fold product $e_{1} \ldots e_{s}$ of edges of $G$ if there is a sequence of vertices $p_{0}, p_{1}, \ldots, p_{2 \ell+1}$ for some $\ell \geq 1$ in $G$ such that

(i) $p_{i} p_{i+1} \in G$ for all $0 \leq i \leq 2 \ell$,

(ii) $p_{0}=u$ and $p_{2 \ell+1}=v$,

(iii) for all $0 \leq j \leq \ell-1, p_{2 j+1} p_{2 j+2}=e_{i}$ for some $i$, and

(iv) for all $k,\left|\left\{j \mid\left\{p_{2 j+1}, p_{2 j+2}\right\}=e_{k}\right\}\right| \leq\left|\left\{t \mid e_{t}=e_{k}\right\}\right|$.

In such case, $p_{0}, p_{1}, \ldots, p_{2 \ell+1}$ is called an even-connection between $u$ and $v$ with respect to $e_{1} \ldots e_{s}$.

Theorem 2.10. [2, Theorems 6.1, 6.5 and 6.7] Let $G$ be a graph with edge ideal $I=I(G)$ and let $s \geq 1$. Let $M=e_{1} \ldots e_{s}$ be a minimal monomial generator of $I^{s}$ where $e_{1}, \ldots, e_{s}$ are some edges of $G$. Then $\left(I^{s+1}: M\right)$ is minimally generated by monomials of degree 2 , and $u v$ is a minimal generator of $\left(I^{s+1}: M\right)$ if and only if either $u v \in G$ or $u$ and $v$ are even-connected with respect to $M$. 
Consequently, if $G$ is a graph and $M$ is a minimal generator of $I(G)^{s}$ for some $s \geq 1$, then $\left(I(G)^{s+1}: M\right)^{\text {pol }}$ is the edge ideal of some associated graph $G^{\prime}$. When $G$ is a gap-free graph, some combinatorial properties of $G^{\prime}$ are summarized in the next result.

Theorem 2.11. [2, Lemmas 6.14 and 6.15] Let $G$ be a gap-free graph with edge ideal $I=I(G)$ and let $e_{1}, \ldots, e_{s}$ be some edges of $G$ where $s \geq 1$. Then the graph $G^{\prime}$ which is associated to $\left(I^{s+1}: e_{1} \ldots e_{s}\right)^{\mathrm{pol}}$ is gap-free as well. Also if $C_{n}=\left(u_{1} \ldots u_{n}\right)$ is a cycle on $n \geq 5$ vertices such that $C_{n}^{c}$ is an induced subgraph of $G^{\prime}$, then $C_{n}^{c}$ is an induced subgraph of $G$ as well.

The next theorem is one of the main graph theoretic tools that will be used in the sequel.

Theorem 2.12. [4, Theorems 2 and 3] Let $G$ be a gap-free graph with no isolated vertices.

(i) If $\omega(G) \geq 3$, then $G$ has a dominating clique on $\omega(G)$ vertices.

(ii) If $w(G)=2$ and $G$ is not bipartite, then $G$ can be obtained from a five-cycle by vertex multiplication.

\section{Colon ideals of powers of edge ideals}

In this section, we first prove some graph theoretic results regarding the structure of (gap, diamond)-free graphs. Then we analyze the colon ideals of the form $\left(I(G)^{s+1}: m\right)$ where $m$ is a minimal monomial generator of $I(G)^{s}$.

Proposition 3.1. Let $H$ be a graph which is obtained from $G$ by multiplying a vertex $v \in V(G)$ by some $k \geq 2$.

(i) $G$ is gap-free if and only if $H$ is gap-free.

(ii) $G$ and $H$ have the same clique number.

(iii) If $G$ is diamond-free, then $H$ is diamond-free if and only if $v$ does not belong to any triangle of $G$.

Proof. Suppose that $v$ is replaced with the independent set $\left\{v_{1}, \ldots, v_{k}\right\}$.

( $i$ ) If $H$ is gap-free, then $G$ is gap-free since $G$ can be considered as an induced subgraph of $H$. Assume for a contradiction $G$ is gap-free but there is a gap between $a b, c d \in H$. Observe that since the vertices $v_{1}, \ldots, v_{k}$ have the same neighbors in $H$, at most one of them belongs to $\{a, b, c, d\}$. Then this gives a gap in $G$.

(ii) Since every clique of $H$ contains at most one vertex of $\left\{v_{1}, \ldots, v_{k}\right\}$, the result follows.

(iii) Suppose that $G$ is diamond-free. If $v$ belongs to a triangle of $G$, then $H$ clearly has an induced diamond. Conversely, suppose that $H$ has an induced diamond with edges $a b, a d, b d, b c, c d$. Since $G$ is diamond-free, without loss of generality we may assume that either $v_{1}=a$ or $v_{1}=d$. If $v_{1}=a$, then $(v b d)$ is a triangle in $G$. If $v_{1}=d$, then $(a v b)$ is a triangle in $G$. 
Lemma 3.2. Let $G$ be a gap-free and diamond-free graph with $\omega(G) \geq 3$ and with no isolated vertices. Then there is a dominating clique $K_{\omega(G)}$ such that every vertex outside of $K_{\omega(G)}$ is adjacent to exactly one vertex of $K_{\omega(G)}$. Moreover the following statements hold.

(i) If $\omega(G) \geq 4$, then $G-K_{\omega(G)}$ is independent and in particular, $G^{c}$ is chordal.

(ii) If $\omega(G)=3$, then for every vertex $x$ of $K_{3}$ the set $N(x) \backslash V\left(K_{3}\right)$ is independent and in particular, $G-$ st $x$ is bipartite.

Proof. Let $K_{\omega(G)}$ be a dominating clique on the vertices $u_{1}, \ldots, u_{\omega(G)}$ as in Theorem 2.12.

Suppose $v$ is a vertex of $G$ which is not in $K_{\omega(G)}$. Assume for a contradiction $v u_{i} \in G$ and $v u_{j} \in G$ for some $i \neq j$. Let $k$ be different from $i$ and $j$. Since $G$ is diamond-free, $u_{k} v \in G$. Since $k$ was arbitrary, it follows that $v$ is connected to all vertices of $K_{\omega(G)}$. Thus $G$ contains a clique with $\omega(G)+1$ vertices, a contradiction.

(i) Let $v$ and $w$ be distinct vertices outside of $K_{\omega(G)}$. Assume for a contradiction $v w \in G$. Suppose $v u_{i} \in G$ and $w u_{j} \in G$ for some $i, j$. Since $\omega(G) \geq 4$, there exist distinct vertices $u_{k}$ and $u_{\ell}$ such that $\ell \neq i, j$ and $k \neq i, j$. We already know that none of $v u_{k}, v u_{\ell}, w u_{k}, w u_{\ell}$ is in $G$. Hence there is a gap between $v w$ and $u_{k} u_{\ell}$, a contradiction.

(ii) Let $x, y$ and $z$ be the vertices of $K_{3}$. Assume for a contradiction $u x, v x, u v \in G$ for some $u, v \notin V\left(K_{3}\right)$. Since $u x \in G, u$ cannot be adjacent to $y$ or $z$. Similarly $v$ cannot be adjacent to $y$ or $z$. Hence there is a gap between $u v$ and $y z$ which is a contradiction.

Remark 3.3. Observe that the complement of a $C_{5}$ is again a cycle on 5 vertices. Therefore a graph is $C_{5}$-free if and only if it is $C_{5}^{c}$-free. We shall use this fact for the rest of the paper without reference.

Lemma 3.4. Suppose $G$ is a gap-free graph.

(i) If $G$ is bipartite, then $G^{c}$ is chordal.

(ii) Let $G$ be connected $\left(C_{5}\right.$, diamond)-free and $\omega(G)=3$. Then either $G=C_{6}^{c}$ or $G^{c}$ is chordal.

Proof. (i) Assume for a contradiction $G$ is bipartite but $G^{c}$ has an induced cycle $C_{n}=$ $\left(x_{1} x_{2} \ldots x_{n}\right)$ where $n \geq 4$. Since $G$ is gap-free, we must have $n \geq 5$. Let $A \cup B$ be a bipartition of $G$ where $x_{2} \in A$. Then $x_{4}, x_{5} \in B$ since $x_{2} x_{4}, x_{2} x_{5} \in G$. As $x_{1} x_{4} \in G$ we get $x_{1} \in A$. Similarly, as $x_{1} x_{3} \in G$, we get $x_{3} \in B$. But then since $x_{3} x_{5}$ is an edge of $G$, the set $B$ is not independent, which is a contradiction.

(ii) Assume for a contradiction $G \neq C_{6}^{c}$ and $G^{c}$ is not chordal. Then $G^{c}$ has an induced $C_{n}=\left(x_{1} \ldots x_{n}\right)$ for some $n \geq 6$.

Case 1: Let $n=6$. Then since $G \neq C_{6}^{c}$ and $G$ is connected, without loss of generality assume that there is a vertex $y \in V(G) \backslash V\left(C_{6}^{c}\right)$ such that $y x_{1}$ is an edge of $G$. Since there is no gap between $y x_{1}$ and $x_{2} x_{6}$, either $y x_{6}$ or $y x_{2}$ must be an edge of $G$. Without loss of generality assume that $y x_{2} \in G$. Since the induced subgraph on the vertices $x_{1}, x_{3}, x_{6}, x_{2}, y$ is not an induced 5-cycle of $G$, we must have either $x_{3} y \in G$ or $x_{6} y \in G$. Without loss 
of generality suppose that $x_{3} y \in G$. Consider the induced subgraph $H$ on the vertices $x_{1}, x_{3}, x_{5}, y$. If $y x_{5} \notin G$, then $H$ is an induced diamond, contradiction. If $y x_{5} \in G$, then $H$ is a clique on 4 vertices, which is again a contradiction as $\omega(G)=3$.

Case 2: Let $n \geq 7$. Then the induced subgraph on the vertices $x_{1}, x_{3}, x_{4}, x_{6}$ is a diamond, contradiction.

Theorem 3.5. Suppose $G$ is both gap-free and diamond-free. Then $\operatorname{reg}(I(G)) \leq 3$.

Proof. If $G$ is triangle-free, then it is cricket-free and thus the result follows from [2, Theorem 3.4]. If $\omega(G) \geq 4$, then the result follows from Lemma 3.2 and Theorem 2.8 . Therefore we may assume that $\omega(G)=3$. We proceed by induction on the number of vertices of $G$. If $G$ is a triangle, then $\operatorname{reg}(I(G))=2$. Let $K_{3}$ be a dominating clique as in Lemma 3.2 and let $x$ be a vertex of $K_{3}$. Since $G-x$ is either triangle-free or have clique number 3 we get $\operatorname{reg}(I(G-x)) \leq 3$ by induction. From Lemma 3.2 and Lemma 3.4 the graph $(G-\text { st } x)^{c}$ is chordal and $\operatorname{reg}(I(G-\operatorname{st} x))=2$ follows from Theorem 2.8. Thus $\operatorname{reg}(I(G)) \leq 3$ using Theorem 2.4,

Lemma 3.6. Let $G$ be a gap-free graph and let $e_{1} \ldots e_{s}$ be an s-fold product of edges. Let $C_{n}=\left(u_{1} \ldots u_{n}\right)$ be a cycle where $n \geq 5$. Suppose $C_{n}^{c}$ is an induced anticycle in the graph which is associated to $\left(I(G)^{s+1}: e_{1} \ldots e_{s}\right)^{\mathrm{pol}}$. Then $e_{i} \cap\left\{u_{1}, \ldots, u_{n}\right\}=\emptyset$ for every $1 \leq i \leq s$.

Proof. Let $G^{\prime}$ be the gap-free graph associated to $\left(I(G)^{s+1}: e_{1} \ldots e_{s}\right)^{\text {pol }}$. First note that by Theorem 2.11, $C_{n}^{c}$ is also an induced anticycle in $G$. Let $e_{i}=x y$ be fixed. Notice that by symmetry, it is enough to consider the following two cases.

Case 1: Suppose $\{x, y\} \cap\left\{u_{1}, \ldots, u_{n}\right\}=\{x\}$. Without loss of generality assume that $x=u_{1}$. Since there is no gap between $x y$ and $u_{2} u_{n}$, either $y u_{2} \in G$ or $y u_{n} \in G$. Without loss of generality suppose $y u_{2} \in G$. Then $u_{2} y x u_{3}$ is an even-connection. Thus $u_{2} u_{3} \in G^{\prime}$ which is a contradiction.

Case 2: Suppose $\{x, y\} \cap\left\{u_{1}, \ldots, u_{n}\right\}=\{x, y\}$. Without loss of generality, assume that $x=u_{1}$. Then $y=u_{i}$ for some $i \neq 2, n$. Since $n \geq 5$, either $i+1<n$ or $i>3$. If $i+1<n$, then $u_{n} y x u_{n-1}$ is an even-connection and $u_{n} u_{n-1} \in G^{\prime}$, a contradiction. If $i>3$, then $u_{2} y x u_{3}$ is an even-connection and $u_{2} u_{3} \in G^{\prime}$, a contradiction.

Lemma 3.7. Let $G$ be a (diamond, gap)-free graph and let $s \geq 1$. For every minimal generator $m$ of $I(G)^{s}$, the graph associated to $\left(I(G)^{s+1}: m\right)^{\text {pol }}$ does not contain induced $C_{n}^{c}$ for all $n \geq 6$.

Proof. Let $G^{\prime}$ be the gap-free graph which is associated to $\left(I(G)^{s+1}: m\right)^{\text {pol }}$ as in Theorem [2.11. Assume for a contradiction $G^{\prime}$ has induced $C_{n}^{c}$ for some $n \geq 6$ where $C_{n}=$ $\left(v_{1} \ldots v_{n}\right)$. From Theorem [2.11, the anticycle $C_{n}^{c}$ is an induced subgraph of $G$. Let $m=e_{1} \ldots e_{s}$ for some edges $e_{1}, \ldots, e_{s}$ of $G$ and let $e_{1}=a b$. From Lemma 3.6 it follows that neither $a$ nor $b$ belongs to $C_{n}$. Since there is no gap between $v_{1} v_{3}$ and $a b$, without loss of generality assume that $v_{1} a \in G$. Notice that $v_{2} b \notin G$ since otherwise $v_{1} a b v_{2}$ is an even-connection and $v_{1} v_{2} \in G^{\prime}$, which is a contradiction. Similarly, $v_{n} b \notin G$ as otherwise $v_{1} a b v_{n}$ would be even-connection and that would require $v_{1} v_{n} \in G^{\prime}$. Since there is no gap 
between $v_{2} v_{n}$ and $a b$, without loss of generality assume that $v_{2} a \in G$. Observe that $v_{3} b \notin G$ as otherwise $v_{2} a b v_{3}$ would be even connection and that would require $v_{2} v_{3} \in G^{\prime}$. Because there is no gap between $v_{3} v_{n}$ and $a b$, either $v_{n} a \in G$ or $v_{3} a \in G$. Without loss of generality, suppose $v_{3} a \in G$. If $a v_{5} \in G$, then the induced subgraph of $G$ on the vertices $v_{2}, v_{3}, v_{5}, a$ is a diamond, which is a contradiction. Otherwise, the induced subgraph of $G$ on the vertices $v_{1}, v_{3}, v_{5}, a$ is a diamond, again a contradiction.

Lemma 3.8. Let $G$ be a gap-free and diamond-free graph with $\omega(G)=3$. Let $K_{3}=(a b c)$ be a dominating clique of $G$.

(i) For all $s \geq 1$ and for all $(s-1)$-fold product $e_{1} \ldots e_{s-1}$ edges of $G$, every induced $C_{5}$ in the graph of $\left(I(G)^{s+1}:(a b) e_{1} \ldots e_{s-1}\right)^{\mathrm{pol}}$ contains at least 2 vertices of $K_{3}$.

(ii) For all $s \geq 1$ and for all $(s-1)$-fold product $e_{1} \ldots e_{s-1}$ edges of $G, \operatorname{reg}\left(\left(I(G)^{s+1}\right.\right.$ : $\left.\left.(a b) e_{1} \ldots e_{s-1}\right)^{\mathrm{pol}}\right)=2$.

Proof. ( $i)$ Let $C_{5}=\left(u_{1} u_{2} u_{3} u_{4} u_{5}\right)$ be an induced cycle of $G^{\prime}$ where $G^{\prime}$ is the graph associated to $\left(I(G)^{s+1}:(a b) e_{1} \ldots e_{s-1}\right)^{\text {pol }}$ as in Theorem 2.11. Then since $G$ is a subgraph of $G^{\prime}$ and $V\left(C_{5}\right) \subseteq V(G)$, the cycle $C_{5}$ is also an induced subgraph of $G$. Keeping Lemma 3.2 in mind, assume for a contradiction $V\left(C_{5}\right) \cap V\left(K_{3}\right)$ has at most one vertex.

Case 1: Suppose that $V\left(C_{5}\right) \cap\{a, b, c\}=\emptyset$. Since no set of 3 vertices of $C_{5}$ is independent, each of $N(a) \backslash V\left(K_{3}\right), N(b) \backslash V\left(K_{3}\right)$ and $N(c) \backslash V\left(K_{3}\right)$ contains at least one vertex of $C_{5}$. Without loss of generality assume that $\left(N(a) \backslash V\left(K_{3}\right)\right) \cap V\left(C_{5}\right)=$ $\left\{u_{5}\right\},\left(N(b) \backslash V\left(K_{3}\right)\right) \cap V\left(C_{5}\right)=\left\{u_{1}, u_{3}\right\}$ and $\left(N(c) \backslash V\left(K_{3}\right)\right) \cap V\left(C_{5}\right)=\left\{u_{2}, u_{4}\right\}$. Then, there is no edge in $G$ that connects $u_{2} u_{3}$ and $a u_{5}$, which is absurd because $G$ is gap-free.

Case 2: Suppose that $\left|V\left(C_{5}\right) \cap\{a, b, c\}\right|=1$. Without loss of generality assume that $a=u_{1} \in V\left(C_{5}\right) \cap\{a, b, c\}$. Then $\left(N(a) \backslash V\left(K_{3}\right)\right) \cap V\left(C_{5}\right)=\left\{u_{2}, u_{5}\right\}$. Without loss of generality we may assume that $u_{4}$ is adjacent to $b$ and $u_{3}$ is adjacent to $c$ in $G$. But then $u_{2} a b u_{4}$ is an even-connection and $u_{2} u_{4} \in G^{\prime}$. This is a contradiction because $C_{5}$ is an induced cycle of $G^{\prime}$.

(ii) Let $G^{\prime}$ be the gap-free graph which is associated to $\left(I(G)^{s+1}:(a b) e_{1} \ldots e_{s-1}\right)^{\text {pol }}$ as in Theorem 2.11, From Fröberg's Theorem and Lemma 3.7 it suffices to show that $G^{\prime}$ has no induced $C_{5}$. Assume for a contradiction $G^{\prime}$ has induced cycle $C_{5}=\left(u_{1} \ldots u_{5}\right)$. From Lemma 3.6 we have $\{a, b\} \cap\left\{u_{1}, \ldots, u_{5}\right\}=\emptyset$. On the other hand, by part $(i)$ we have $\left|\{a, b, c\} \cap\left\{u_{1}, \ldots, u_{5}\right\}\right| \geq 2$ which is a contradiction.

\section{Regularity of powers of (diamond, gap)-free graphs}

The authors of [1] classified all imperfect $P_{5}$-free and diamond-free graphs based on a special family of graphs. Our main result Theorem 4.9 will be based on this classification. To state Arbib and Mosca's theorem, we need some notation first. All the graphs in [1, Fig. 3] contain an induced $C_{5}=\left(u_{0} u_{1} u_{2} u_{3} u_{4}\right)$. Let $D_{k}$ denote the set of points at distance $k$ from this $C_{5}$. 
Theorem 4.1. [1, Theorem 1.6] Any connected ( $P_{5}$, diamond)-free graph that properly contains an induced $C_{5}$ can be obtained from $C_{5}$ or from a graph among those of [1, Fig. 3] by multiplying some $v \in D_{1} \cup C_{5}$ and/or substituting a $P_{3}$-free graph for some $v \in D_{2}$.

Since any gap-free graph is $P_{5}$-free we obtain the following corollary.

Corollary 4.2. Any connected (gap, diamond)-free graph that properly contains an induced $C_{5}$ is either one of the graphs in Fig. 1 or it can be obtained from $C_{5}$ or from a graph among those of Fig. 1 by multiplying some vertices which do not belong to any triangles.

Proof. First note that multiplying a vertex of a triangle by $k>1$ yields a diamond. Observe that $G_{10}$ and $G_{0}$ are the only graphs in [1, Fig. 3] that contain vertices of distance 2 from the induced $C_{5}=\left(u_{0} u_{1} u_{2} u_{3} u_{4}\right)$. Observe that in Fig. 2 there is a gap between the edges $u_{0} a_{0}$ and $b_{0} b_{2}$ of $G_{4}$. Therefore a (gap, diamond)-free graph cannot be obtained from $G_{4}$ by Proposition 3.1. Let $H$ be a graph that contains at least one edge. We claim that substituting $H$ for $y$ in $G_{10}$ or $G_{0}$ yields a gap. Indeed, if $y_{1} y_{2}$ is an edge of $H$, then there is a gap between $y_{1} y_{2}$ and $u_{1} u_{2}$.

Remark 4.3. Let $H$ be a graph and let $u$ be a vertex of $H$. Let $G$ be obtained from $H$ by multiplying the vertex $u$ by the independent set $U$. If $C_{n}$ is an induced cycle of $G$ where $n \geq 5$, then $C_{n}$ contains at most one vertex from $U$. In particular, if $H$ is $C_{n}$-free where $n \geq 5$, then so is $G$.

The proof of the next lemma is computer aided. We used Maple to list the induced 5-cycles of graphs in Fig. 1.

Lemma 4.4. Let $G$ be a (gap, diamond)-free graph which is obtained from a graph among the graphs $G_{1}, G_{2}, \ldots, G_{9}$ in Fig. 1 by vertex multiplication. Let $C_{5}$ be an induced subgraph of $G$ and $e=\{a, b\}$ be an edge of $G$ such that $e \cap V\left(C_{5}\right)=\emptyset$. Then at least one of the following statements holds.

(i) $e \in E\left(K_{3}\right)$ for some dominating clique $K_{3}$ of $G$.

(ii) There exists distinct $u, v \in V\left(C_{5}\right)$ such that $a u \in G, b v \in G$ and $u v \notin C_{5}$.

Proof. Using Corollary 4.2 and keeping Remark 4.3 in mind, we consider cases.

Case 1: Let $G$ be obtained from $G_{1}$ by replacing the vertices $u_{0}, u_{1}, u_{4}$ respectively with the independent sets $U_{0}, U_{1}, U_{4}$. Since $\left(u_{0} \ldots u_{4}\right)$ is the only induced 5-cycle of $G_{1}$, we may assume that $C_{5}=\left(u_{0}^{1} u_{1}^{1} u_{2} u_{3} u_{4}^{1}\right)$ for some $u_{i}^{1} \in U_{i}$. Since $e$ does not intersect $C_{5}$, by the symmetry of the graph we may assume $e=u_{0}^{2} u_{4}^{2}$ or $e=u_{0}^{2} a_{0}$ for some $u_{i}^{2} \in U_{i} \backslash\left\{u_{i}^{1}\right\}$. In both cases, one vertex of $e$ is adjacent to $u_{3}$ and the other one is adjacent to $u_{1}^{1}$. Since $u_{1}^{1} u_{3} \notin C_{5}$, the statement (ii) holds.

Case 2: Suppose that $G$ is obtained from $G_{2}$ by replacing the vertex $u_{1}$ with $\left\{u_{1}^{1}, \ldots, u_{1}^{k}\right\}$. Since $\left(u_{0} \ldots u_{4}\right)$ is the only induced 5 -cycle of $G_{2}$, we may assume that $C_{5}=\left(u_{0} u_{1}^{1} u_{2} u_{3} u_{4}\right)$. But then every edge of $G$ intersects $C_{5}$. 
Case 3: Suppose that $G$ is obtained from $G_{3}$ by multiplying $u_{0}, u_{2}, u_{3}, u_{4}$. For each $i=0,2,3,4$ let $u_{i}$ be replaced with $\left\{u_{i}^{1}, \ldots, u_{i}^{k_{i}}\right\}$ in $G$. Suppose $e$ does not belong to the dominating clique $\left(b_{0} b_{2} u_{1}\right)$. Since the induced 5-cycles of $G_{3}$ are $\left(u_{0} \ldots u_{4}\right),\left(b_{0} u_{1} u_{2} u_{3} u_{4}\right)$ and $\left(b_{2} u_{1} u_{0} u_{4} u_{3}\right)$, the symmetry of the graph allows us to consider the following cases.

Case 3.1: Suppose $C_{5}=\left(b_{0} u_{1} u_{2}^{1} u_{3}^{1} u_{4}^{1}\right)$. Then $e$ can have forms $u_{0}^{i} u_{4}^{j}, u_{3}^{i} u_{4}^{j}, u_{3}^{i} u_{2}^{j}$ or $u_{3}^{i} b_{2}$. If $e=u_{0}^{i} u_{4}^{j}$, then (ii) holds because the endpoints of $e$ are respectively adjacent to $u_{1}$ and $u_{3}^{1}$ and $u_{1} u_{3}^{1} \notin C_{5}$. Similarly, if $e=u_{3}^{i} u_{4}^{j}$, then the endpoints of $e$ are respectively adjacent to $u_{2}^{1}$ and $b_{0}$ but $u_{2}^{1} b_{0} \notin C_{5}$. Lastly, if $e=u_{3}^{i} u_{2}^{j}$ or $e=u_{3}^{i} b_{2}$ then the endpoints of $e$ are respectively adjacent to $u_{4}^{1}$ and $u_{1}$ but $u_{1} u_{4}^{1} \notin C_{5}$.

Case 3.2: Suppose $C_{5}=\left(u_{0}^{1} u_{1} u_{2}^{1} u_{3}^{1} u_{4}^{1}\right)$. Notice that if $e=u_{p}^{i} u_{q}^{j}$ for some $u_{p} u_{q} \in C_{5}$, then $(i i)$ clearly holds. By the symmetry of the graph, we may assume that $e=b_{0} u_{4}^{i}$. Then $u_{3}^{1} u_{4}^{i} \in G, b_{0} u_{1} \in G$ but $u_{1} u_{3}^{1} \notin G$ and $(i i)$ is satisfied.

Case 4: Suppose that $G$ is obtained from $G_{5}$ by multiplying the vertices $u_{1}, u_{4}$. Observe that $K_{3}=\left(b_{1} b_{4} u_{0}\right)$ is a dominating clique and every induced 5-cycle of $G$ contains the vertices $u_{0}, u_{2}, u_{3}$. It follows that every edge of $G$ either belongs to $K_{3}$ or intersects $C_{5}$.

Case 5: Suppose that $G$ is obtained from $G_{6}$ by multiplying the vertices $u_{1}, u_{4}, b_{2}$. Suppose that $u_{1}$ and $b_{2}$ are respectively replaced with the independent sets $U$ and $B$. Note that $\left(b_{1} b_{4} u_{0}\right)$ and $\left(a_{0} u_{2} u_{3}\right)$ are dominating cliques of $G$. Suppose that $e$ does not belong to these dominating triangles. Note that every induced 5-cycle of $G$ contains both $u_{0}$ and $u_{3}$. So, $e$ contains neither $u_{0}$ nor $u_{3}$. Then $e$ can take the forms $u u_{2}, u b, b_{1} u_{2}$ or $b_{1} b$ for some $u \in U, b \in B$. In each case, one vertex of $e$ is adjacent to $u_{0}$ and the other one is adjacent to $u_{3}$. But since $u_{0} u_{3} \notin C_{5}$, the statement (ii) holds.

Case 6: Suppose that $G$ is obtained from $G_{7}$ by multiplying the vertices $u_{1}, b_{3}, b_{4}$. Suppose that $b_{3}$ and $b_{4}$ are respectively replaced with the independent sets $B_{3}$ and $B_{4}$. Note that $\left(a_{2} u_{0} u_{4}\right)$ and $\left(a_{0} u_{2} u_{3}\right)$ are dominating cliques of $G$. Suppose that $e$ does not belong to these dominating triangles. Note that every induced 5-cycle of $G$ contains both $u_{0}$ and $u_{2}$. So, $e$ contains neither $u_{0}$ nor $u_{2}$. Then $e$ can take the forms $u_{3} u_{4}, u_{3} b_{4}^{\prime}, b_{3}^{\prime} u_{4}$ or $b_{3}^{\prime} b_{4}^{\prime}$ for some $b_{3}^{\prime} \in B_{3}, b_{4}^{\prime} \in B_{4}$. In each case, one vertex of $e$ is adjacent to $u_{0}$ and the other one is adjacent to $u_{2}$. But since $u_{0} u_{2} \notin C_{5}$, the statement (ii) holds.

Case 7: Suppose that $G$ is obtained from $G_{8}$ by multiplying the vertices $b_{2}, b_{4}, u_{1}$. Suppose that $u_{1}$ and $b_{2}$ are respectively replaced with the independent sets $U$ and $B$. Note that $\left(a_{2} u_{0} u_{4}\right)$ and $\left(a_{0} u_{2} u_{3}\right)$ are dominating cliques of $G$. Suppose that $e$ does not belong to these dominating triangles. Note that every induced 5-cycle of $G$ contains both $u_{0}$ and $u_{3}$. So, $e$ contains neither $u_{0}$ nor $u_{3}$. Then $e$ can take the forms $u b, u u_{2}, a_{2} u_{2}$ or $a_{2} b$ for some $b \in B, u \in U$. In each case, one vertex of $e$ is adjacent to $u_{0}$ and the other one is adjacent to $u_{3}$. But since $u_{0} u_{3} \notin C_{5}$, the statement $(i i)$ holds.

Case 8: Since every vertex of $G_{9}$ belongs to a triangle, $G_{9}$ does not generate any other graphs. Let $e$ be an edge which does not belong to any of the dominating triangles $\left(a_{0} u_{2} u_{3}\right),\left(u_{0} a_{2} u_{4}\right)$ and $\left(b_{0} b_{2} u_{1}\right)$. Examining all 5 -cycles of $G_{9}$, we see that there are 3 cases to consider.

Case 8.1: Suppose $C_{5}$ contains $a_{0}, a_{2}$ and $b_{0}$. Then $e$ is equal to one of $u_{3} u_{4}, u_{3} b_{2}, u_{2} u_{1}$ or $u_{0} u_{1}$. If $e$ is equal to $u_{3} u_{4}$ or $u_{3} b_{2}$, then one vertex of $e$ is adjacent to $a_{0}$ and the other 
one is adjacent to $a_{2}$. But $a_{0} a_{2} \notin C_{5}$ so $(i i)$ holds. Otherwise, one vertex of $e$ is adjacent to $a_{2}$ and the other one is adjacent to $b_{0}$ and $b_{0} a_{2} \notin C_{5}$.

Case 8.2: Suppose $C_{5}$ contains $b_{2}, u_{0}$ and $u_{3}$. Then $e$ is equal to one of $b_{0} u_{4}, b_{0} a_{0}, a_{2} u_{2}$ or $u_{1} u_{2}$. If $e$ is equal to $b_{0} u_{4}$ or $b_{0} a_{0}$, then one vertex of $e$ is adjacent to $b_{2}$ and the other one is adjacent to $u_{0}$. But $b_{2} u_{0} \notin C_{5}$ so $(i i)$ holds. Otherwise, one vertex of $e$ is adjacent to $u_{0}$ and the other one is adjacent to $u_{3}$ and $u_{0} u_{3} \notin C_{5}$.

Case 8.3: Suppose $C_{5}$ contains $u_{1}, u_{2}$ and $u_{4}$. Then $e$ is equal to one of $b_{0} a_{0}, u_{0} a_{0}, b_{2} a_{2}$ or $b_{2} u_{3}$. If $e$ is equal to $b_{0} a_{0}$ or $u_{0} a_{0}$, then one vertex of $e$ is adjacent to $u_{4}$ and the other one is adjacent to $u_{2}$. But $u_{2} u_{4} \notin C_{5}$ so $(i i)$ holds. Otherwise, one vertex of $e$ is adjacent to $u_{1}$ and the other one is adjacent to $u_{4}$. But $u_{1} u_{4} \notin C_{5}$, completing the proof.

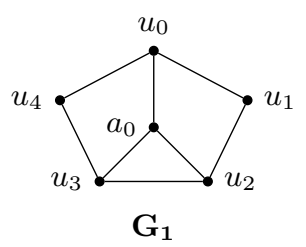

$\mathbf{G}_{1}$
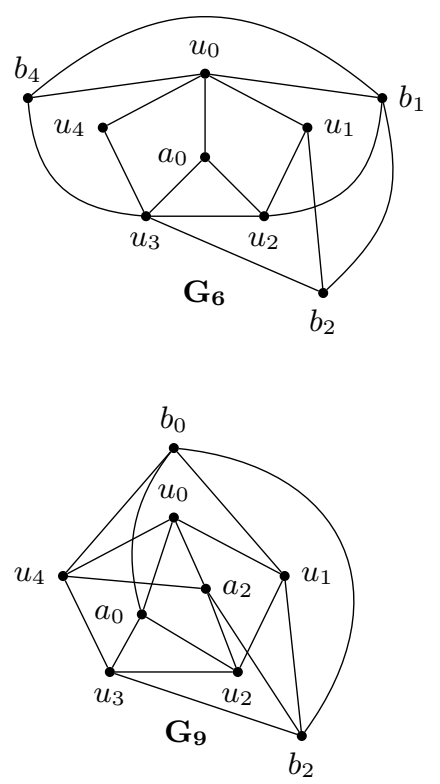

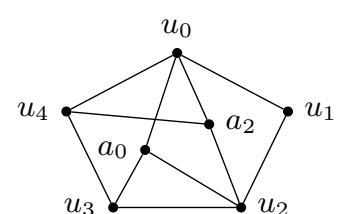

$\mathbf{G}_{2}$

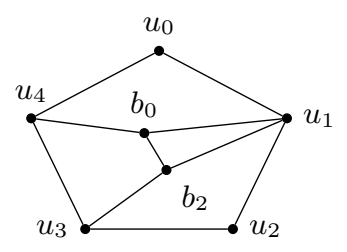

$\mathrm{G}_{3}$

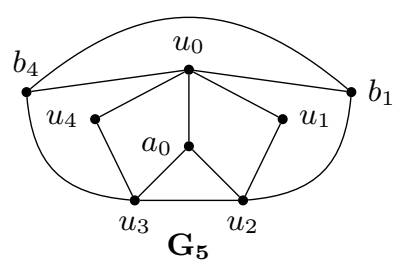

G
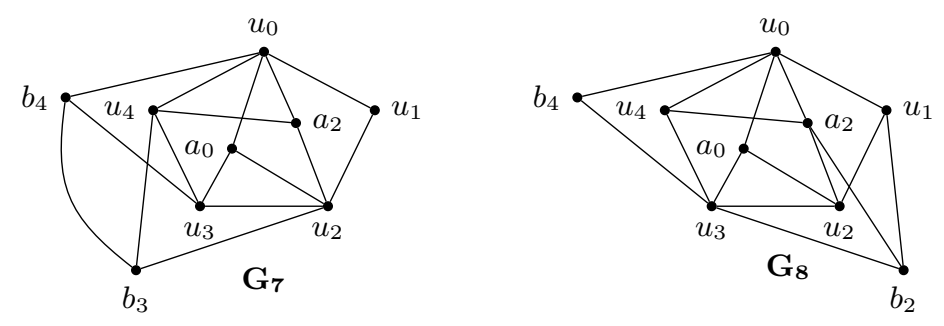

Figure 1: The list of graphs from which every (gap, diamond)-free graph in Corollary 4.2 can be generated by vertex multiplication. 


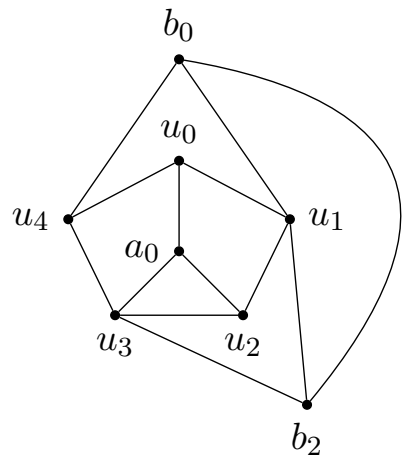

Figure 2: The graph $\mathbf{G}_{\mathbf{4}}$ in [1, Fig. 3] which is not gap-free.

Theorem 4.5. Let $G$ be a (gap, diamond)-free graph which is obtained from a graph among the graphs $G_{1}, G_{2}, \ldots, G_{9}$ in Fig 1 by vertex multiplication. Then $\operatorname{reg}\left(I(G)^{s+1}\right)=2 s+2$ for all $s \geq 1$.

Proof. Since each of the graphs $G_{1}, G_{2}, \ldots, G_{9}$ contains an induced $C_{5}$ and a triangle, all of them have clique number 3 by Lemma 3.2(i). Let $s$ be fixed and let $m=e_{1} \ldots e_{s}$ be a monomial generator of $I(G)^{s}$ for some edges $e_{1}:=a b, e_{2}, \ldots, e_{s}$ of $G$. We claim that $\operatorname{reg}\left(I(G)^{s+1}: m\right) \leq 2$ and then proof follows from Theorem 3.5 and Theorem 2.7. Note that if $e_{1}$ is an edge of a dominating $K_{3}$ of $G$, then the proof follows from Lemma 3.8(ii). So, let us assume that $e_{1}$ does not belong to a dominating $K_{3}$. Let $G^{\prime}$ be the gap-free graph which is associated to $\left(I(G)^{s+1}: m\right)^{\text {pol }}$ as in Theorem 2.11. From Fröberg's theorem and Lemma 3.7, it suffices to show that $G^{\prime}$ has no induced cycle on 5 vertices. Assume for a contradiction $G^{\prime}$ has an induced $C_{5}$. Then $C_{5}$ is an induced subgraph of $G$ by Theorem 2.11, We know that $e_{1} \cap V\left(C_{5}\right)=\emptyset$ from Lemma 3.6. Now, from Lemma 4.4 there exists distinct $u, v \in V\left(C_{5}\right)$ such that $a u \in G, b v \in G$ and $u v \notin C_{5}$. Then $u a b v$ is an even connection with respect to $e_{1} \ldots e_{s}$ and $u v \in G^{\prime}$. But then since $C_{5}$ is an induced subgraph of $G^{\prime}$ we get $u v \in C_{5}$, which is a contradiction completing the proof.

The proof of the theorem above does not work for (gap,diamond)-free graphs which are obtained from $G_{0}$ or $G_{10}$ in Fig. 1. Using Macaulay 2, one can see $\operatorname{reg}\left(I\left(G_{0}\right)^{2}: y a_{2}\right)=3$ and $\operatorname{reg}\left(I\left(G_{10}\right)^{2}: a_{0} y\right)=3$.

Proposition 4.6. If $G$ is a (gap, diamond)-free graph with $I=I(G)$ which can be generated from $G_{10}$ in Fig. 1 , then $\operatorname{reg}\left(I^{s+1}\right)=2 s+2$ for all $s \geq 1$.

Proof. First note that from Corollary 4.2 the graph $G$ is obtained from $G_{10}$ by multiplying some of the vertices $y, u_{0}, u_{1}, u_{4}$. Let $s \geq 1$ be fixed and let vertices $y, u_{0}, u_{1}, u_{4}$ of $G_{10}$ be respectively replaced by the independent sets $\left\{y_{1}, \ldots, y_{k}\right\},\left\{u_{0}^{1}, \ldots, u_{0}^{m_{0}}\right\},\left\{u_{1}^{1}, \ldots, u_{1}^{m_{1}}\right\}$ and $\left\{u_{4}^{1}, \ldots, u_{4}^{m_{4}}\right\}$ in $G$. We first show that if $e_{1} \ldots e_{s}$ is an $s$-fold product of edges of $G$ with the property that there exists $1 \leq i \leq s$ such that for all $1 \leq j \leq k, y_{j} \nmid e_{i}$, then the ideal $\left(I^{s+1}: e_{1} \ldots e_{s}\right)$ has regularity 2 . To this end, suppose that $y_{j} \nmid e_{1}$ for all $j$. 
From Theorems 2.1, 2.8, 2.11 and Lemma 3.7, it suffices to show that the graph $G^{\prime}$ which is associated to $\left(I^{s+1}: e_{1} \ldots e_{s}\right)^{\text {pol }}$ is $C_{5}$-free. Assume for a contradiction $G^{\prime}$ has induced $C_{5}$. Then from Theorem [2.11, $C_{5}$ is also an induced subgraph of $G$. Recalling Remark 4.3, observe that $C_{5}$ must contain both $u_{2}$ and $u_{3}$. Therefore, without loss of generality, we may assume that $C_{5}=\left(u_{0}^{1} u_{1}^{1} u_{2} u_{3} u_{4}^{1}\right)$. From Lemma 3.6 and the symmetry of the graph, we may assume that $e_{1}=u_{0}^{2} u_{4}^{2}$ or $e_{1}=u_{0}^{2} a_{0}$. If $e_{1}=u_{0}^{2} u_{4}^{2}$, then $u_{3} u_{4}^{2} u_{0}^{2} u_{1}^{1}$ is an even-connection between $u_{3}$ and $u_{1}^{1}$ with respect to $e_{1} \ldots e_{s}$ and $u_{3} u_{1}^{1} \in G^{\prime}$, which is a contradiction because $C_{5}$ is an induced subgraph of $G^{\prime}$. Similarly, if $e_{1}=u_{0}^{2} a_{0}$, then $u_{1}^{1} u_{0}^{2} a_{0} u_{3}$ is an even-connection between $u_{1}^{1}$ and $u_{3}$ with respect to $e_{1} \ldots e_{s}$ and $u_{1}^{1} u_{3} \in G^{\prime}$, a contradiction. This completes the proof of our claim.

Consider the order $e_{1}>\cdots>e_{r}>e_{r+1}>\cdots>e_{r+k}$ on the edges of $G$ where $e_{r+j}=a_{0} y_{j}$ for all $j=1, \ldots, k$. Let $M_{1}>\cdots>M_{z}>M_{z+1}>\cdots>M_{z+t}$ be the order on the minimal monomial generators of $I^{s}$ induced by the order on the edges as in Definition 2.5. Let $z$ be the largest index such that $M_{z}$ has an expression $M_{z}=e_{i_{1}} \ldots e_{i_{s}}$ such that $y_{j} \nmid e_{i_{1}}$ for all $1 \leq j \leq k$. Observe that $M_{z+1}, \ldots, M_{z+t}$ all have unique $s$-fold product expressions up to the permutation of the edges in the product. It follows from the previously proved claim that

$$
\operatorname{reg}\left(I^{s+1}: M_{i}\right)=2 \text { for all } i=1, \ldots, z .
$$

Then repeated use of Lemma 2.2. Theorem 2.6 and Theorem 2.3 yields

$$
\begin{aligned}
\operatorname{reg}\left(I^{s+1}\right) \leq & \max \left\{\operatorname{reg}\left(\left(I^{s+1}: M_{1}\right)\right)+2 s, \operatorname{reg}\left(I^{s+1}, M_{1}\right)\right\} \\
\leq & \max \left\{2+2 s, \operatorname{reg}\left(\left(I^{s+1}, M_{1}\right): M_{2}\right)+2 s, \operatorname{reg}\left(\left(I^{s+1}, M_{1}, M_{2}\right)\right)\right\} \\
= & \max \left\{2+2 s, \operatorname{reg}\left(\left(\left(I^{s+1}: M_{2}\right), \text { some variables }\right)\right)+2 s, \operatorname{reg}\left(\left(I^{s+1}, M_{1}, M_{2}\right)\right)\right\} \\
= & \max \left\{2+2 s, \operatorname{reg}\left(\left(I^{s+1}, M_{1}, M_{2}\right)\right)\right\} \\
& \vdots \\
\leq & \max \left\{2+2 s, \operatorname{reg}\left(I^{s+1}, M_{1}, \ldots, M_{z}\right)\right\} .
\end{aligned}
$$

In order to repeat this process, it remains to show that for all $1 \leq i \leq t$,

$$
\operatorname{reg}\left(\left(I^{s+1}, M_{1}, \ldots, M_{z}, \ldots, M_{z+i-1}\right): M_{z+i}\right) \leq 2 .
$$

To this end, let $1 \leq i \leq t$ be fixed. Then $M_{z+i}=\left(y_{j_{1}} a_{0}\right)^{\alpha_{1}}\left(y_{j_{2}} a_{0}\right)^{\alpha_{2}} \ldots\left(y_{j_{q}} a_{0}\right)^{\alpha_{q}}$ for some $\alpha_{1}, \ldots, \alpha_{q}>0$ and $k \geq j_{1}>j_{2}>\cdots>j_{q} \geq 1$. Observe that $\left(I^{s+1}: M_{z+i}\right)=I$ and using Theorem 2.6 we obtain

$$
\left(\left(I^{s+1}, M_{1}, \ldots, M_{z}, \ldots, M_{z+i-1}\right): M_{z+i}\right)=(I, \text { some variables }) .
$$

Notice that every variable $x \in\left\{u_{0}^{1}, u_{0}^{2}, \ldots, u_{0}^{m_{0}}, u_{2}, u_{3}\right\}$ belongs to the variable generators of the ideal above. Indeed, for every such $x$, we have

$$
M_{z+i-p}=\left(x a_{0}\right)\left(y_{j_{1}} a_{0}\right)^{\alpha_{1}-1}\left(y_{j_{2}} a_{0}\right)^{\alpha_{2}} \ldots\left(y_{j_{q}} a_{0}\right)^{\alpha_{q}}
$$


for some $p>0$. Therefore we obtain

$$
\left(\left(I^{s+1}, M_{1}, \ldots, M_{z}, \ldots, M_{z+i-1}\right): M_{z+i}\right)=\left(I \text {, some variables, } u_{0}^{1}, u_{0}^{2}, \ldots, u_{0}^{m_{0}}, u_{2}, u_{3}\right) .
$$

Let $H$ be the graph with edge ideal $I(H)=\left(y_{1} a_{0}, \ldots, y_{k} a_{0}\right)$. Then

$$
\text { (I, some variables, } \left.u_{0}^{1}, u_{0}^{2}, \ldots, u_{0}^{m_{0}}, u_{2}, u_{3}\right)=(I(H) \text {, some variables }) \text {. }
$$

Since $H^{c}$ is chordal, from Fröberg's Theorem $\operatorname{reg}(I(H))=2$ and then Eq. (2) follows from Lemma 2.2, Now we have

$$
\operatorname{reg}\left(I^{s+1}\right) \leq \max \left\{2+2 s, \operatorname{reg}\left(I^{s+1}, I^{s}\right)\right\}=\max \left\{2+2 s, \operatorname{reg}\left(I^{s}\right)\right\} .
$$

Hence the result follows by induction and Theorem 3.5 .

Since the monomial ideals are polarized by their generators, the following follows from the construction.

Remark 4.7. If $I \subseteq S$ is a monomial ideal and $J \subseteq S$ is a square-free monomial ideal, then $(I+J)^{\mathrm{pol}}=I^{\mathrm{pol}}+J$.

Proposition 4.8. If $G$ is a (gap,diamond)-free graph with $I=I(G)$ which can be generated from $G_{0}$ in Fig. 1, then $\operatorname{reg}\left(I^{s+1}\right)=2 s+2$ for all $s \geq 1$.

Proof. First note that from Corollary 4.2 the graph $G$ is obtained from $G_{0}$ by multiplying some of the vertices $u_{1}, y$. Let $s \geq 1$ be fixed and suppose that the vertices $u_{1}$ and $y$ of $G_{0}$ are respectively replaced by the independent sets $U=\left\{u_{1}^{1}, \ldots, u_{1}^{m}\right\}$ and $Y=\left\{y_{1}, \ldots, y_{k}\right\}$ in $G$. We claim that if $e_{1} \ldots e_{s}$ is an $s$-fold product of edges of $G$ with the property that there exists $1 \leq i \leq s$ such that $x \nmid e_{i}$ for all $x \in U \cup Y$, then $\operatorname{reg}\left(I^{s+1}: e_{1} \ldots e_{s}\right)=2$.

To this end, suppose that $x \nmid e_{1}$ for all $x \in U \cup Y$. From Theorems 2.1, 2.8, 2.11] and Lemma 3.7, it suffices to show that the graph $G^{\prime}$ which is associated to $\left(I^{s+1}: e_{1} \ldots e_{s}\right)^{\text {pol }}$ is $C_{5}$-free. Assume for a contradiction $G^{\prime}$ has induced $C_{5}$. Then $C_{5}$ is also an induced subgraph of $G$ because of Theorem 2.11. Observe that $\left(y a_{2} u_{4} u_{3} a_{0}\right)$ and $\left(u_{0} u_{1} u_{2} u_{3} u_{4}\right)$ are the only induced 5-cycles of $G_{0}$. Because of Remark 4.3 we may assume without loss of generality that $C_{5}=\left(y_{1} a_{2} u_{4} u_{3} a_{0}\right)$ or $C_{5}=\left(u_{0} u_{1}^{1} u_{2} u_{3} u_{4}\right)$. If $C_{5}=\left(y_{1} a_{2} u_{4} u_{3} a_{0}\right)$, then by Lemma 3.6, $e_{1}$ does not intersect any of $a_{2}, u_{4}, u_{3}, a_{0}$ and therefore $e_{1}$ contains a vertex from $U$, which is a contradiction. Similarly, if $C_{5}=\left(u_{0} u_{1}^{1} u_{2} u_{3} u_{4}\right)$, then $e_{1}$ does not intersect any of $u_{0}, u_{2}, u_{3}, u_{4}$ and therefore $e_{1}$ contains a vertex from $Y$, which is a contradiction. This completes the proof of our claim.

Let $e_{1}, \ldots, e_{r}$ be the edges of $G$ which do not contain any vertex from $U \cup Y$. Consider the order

$$
\begin{aligned}
e_{1}>e_{2}>\cdots>e_{r}>y_{1} a_{0}>y_{1} a_{2}>y_{2} a_{0}>y_{2} a_{2}>\cdots>y_{k} a_{0}>y_{k} a_{2} \\
>u_{1}^{1} u_{0}>u_{1}^{1} u_{2}>u_{1}^{2} u_{0}>u_{1}^{2} u_{2}>\cdots>u_{1}^{m} u_{0}>u_{1}^{m} u_{2}
\end{aligned}
$$

on the edges of $G$. Consider the order $M_{1}>\cdots>M_{z}>M_{z+1}>\cdots>M_{z+t}$ on the minimal monomial generators of $I^{s}$ induced by the order on the edges as in Definition 2.5. 
Let $z$ be the largest index such that $M_{z}$ has an expression $M_{z}=e_{i_{1}} \ldots e_{i_{s}}$ such that $x \nmid e_{i_{1}}$ for all $x \in Y \cup U$. It follows from the previously proved claim that

$$
\operatorname{reg}\left(I^{s+1}: M_{i}\right)=2 \text { for all } i=1, \ldots, z .
$$

Then repeated use of Lemma 2.2. Theorem 2.6 and Theorem 2.3 yields

$$
\begin{aligned}
\operatorname{reg}\left(I^{s+1}\right) \leq & \max \left\{\operatorname{reg}\left(\left(I^{s+1}: M_{1}\right)\right)+2 s, \operatorname{reg}\left(\left(I^{s+1}, M_{1}\right)\right)\right\} \\
\leq & \max \left\{2+2 s, \operatorname{reg}\left(\left(I^{s+1}, M_{1}\right): M_{2}\right)+2 s, \operatorname{reg}\left(\left(I^{s+1}, M_{1}, M_{2}\right)\right)\right\} \\
= & \max \left\{2+2 s, \operatorname{reg}\left(\left(\left(I^{s+1}: M_{2}\right), \operatorname{some} \text { variables }\right)\right)+2 s, \operatorname{reg}\left(\left(I^{s+1}, M_{1}, M_{2}\right)\right)\right\} \\
= & \max \left\{2+2 s, \operatorname{reg}\left(\left(I^{s+1}, M_{1}, M_{2}\right)\right)\right\} \\
& \vdots \\
\leq & \max \left\{2+2 s, \operatorname{reg}\left(I^{s+1}, M_{1}, \ldots, M_{z}\right)\right\} .
\end{aligned}
$$

In order to repeat this process, it remains to show that for all $1 \leq i \leq t$,

$$
\operatorname{reg}\left(J_{i}\right):=\operatorname{reg}\left(\left(I^{s+1}, M_{1}, \ldots, M_{z}, \ldots, M_{z+i-1}\right): M_{z+i}\right) \leq 2 .
$$

Let $1 \leq i \leq t$ be fixed. Then $M_{z+i}$ has a maximal expression where the exponent of $x w$ is non-zero for some $x \in U \cup Y$ and $x w \in G$. Let $N(w) \backslash(U \cup Y)=\left\{w_{1}, \ldots, w_{\tau}\right\}$. Then for every $1 \leq \kappa \leq \tau$ we have $M_{z+i} w_{\kappa} / x>M_{z+i}$ since $w w_{\kappa} \in G$ and $w, w_{\kappa} \notin U \cup Y$. Therefore from Theorem 2.1, Theorem 2.6 and Remark 4.7 it follows that

$$
\begin{aligned}
\operatorname{reg}\left(J_{i}\right) & =\operatorname{reg}\left(\left(\left(I^{s+1}: M_{z+i}\right), w_{1}, \ldots, w_{\tau}, \text { some variables }\right)\right) \\
& =\operatorname{reg}\left(\left(\left(I^{s+1}: M_{z+i}\right)^{\text {pol }}, w_{1}, \ldots, w_{\tau}, \text { some variables }\right)\right)
\end{aligned}
$$

Let $G^{\prime}$ be the gap-free graph which is associated to $\left(I^{s+1}: M_{z+i}\right)^{\text {pol }}$ as in Theorem [2.11. Using Lemma 2.2] it suffices to show that $\operatorname{reg}\left(\left(I\left(G^{\prime}\right), w_{1}, \ldots, w_{\tau}\right)\right) \leq 2$. If $\left(I\left(G^{\prime}\right), w_{1}, \ldots, w_{\tau}\right)$ is generated in degree 1 , we have nothing to show. So, let us assume that $\left(\left(I\left(G^{\prime}\right), w_{1}, \ldots, w_{\tau}\right)=I(H)\right.$ where $H=G^{\prime}-\left\{w_{1}, \ldots, w_{\tau}\right\}$ has at least one edge. By Fröberg's theorem and Lemma 3.7, it remains to show that $H$ is $C_{5}$-free. Assume for a contradiction $C_{5}$ is an induced cycle of $H$. Then $C_{5}$ is an induced cycle of $G^{\prime}$ since $H$ is an induced subgraph of $G^{\prime}$. From Theorem 2.11 it follows that $C_{5}$ is an induced cycle of $G$. Observe that every induced 5-cycle of $G_{0}$ contains both $u_{3}$ and $u_{4}$. Therefore $C_{5}$ contains both $u_{3}$ and $u_{4}$ by Remark 4.3. If $w=a_{0}$ or $w=u_{2}$, then $u_{3} \notin V(H)$, and $u_{3} \notin V\left(C_{5}\right)$, a contradiction. Similarly, if $w=a_{2}$ or $w=u_{0}$, then $u_{4} \notin V(H)$, and $u_{4} \notin V\left(C_{5}\right)$, a contradiction.

Now we have

$$
\operatorname{reg}\left(I^{s+1}\right) \leq \max \left\{2+2 s, \operatorname{reg}\left(I^{s+1}, I^{s}\right)\right\}=\max \left\{2+2 s, \operatorname{reg}\left(I^{s}\right)\right\} .
$$

Hence the result follows by induction and Theorem 3.5. 
Finally, we prove the main result of this paper.

Theorem 4.9. If $G$ is a (gap, diamond)-free graph, then $\operatorname{reg}\left(I(G)^{s}\right)=2 s$ for all $s \geq 2$.

Proof. We may assume $G$ is a connected gap-free and diamond-free graph since removal of isolated vertices does not change the edge ideal. If $\omega(G) \geq 4$, then the proof follows from Theorem 2.8, Lemma 3.2 and [8, Theorem 3.2]. If $\omega(G)<3$, then $G$ is cricket-free and the result follows from [2, Theorem 6.17]. Therefore let us assume that $\omega(G)=3$. Note that $C_{6}^{c}$ is cricket-free since $C_{6}^{c}$ has no vertex with 4 neighbors. If $G$ is $C_{5}$-free, then the result follows from combining Theorem [2.8, Lemma 3.4, [2, Theorem 6.17] and [8, Theorem 3.2]. Therefore let us assume that $G$ contains an induced cycle on 5 vertices. If $G$ is obtained from $C_{5}$ by multiplying vertices, then $\omega(G)<3$ by Proposition 3.1. Otherwise, the result follows from combining Corollary 4.2, Theorem 4.5, Proposition 4.6 and Proposition 4.8.

\section{References}

[1] C. Arbib, R. Mosca, On ( $P_{5}$, diamond)-free graphs, Discrete Math. 250 (2002), no. 1-3, $1-22$.

[2] A. Banerjee, The regularity of powers of edge ideals, J. Algebraic Combin. 41 (2015), no. $2,303-321$.

[3] S. Beyarslan, H. T. Hà, T. N. Trung, Regularity of powers of forests and cycles, J. Algebraic Combin. 42 (2015), no. 4, 1077-1095.

[4] F. R. K. Chung, A. Gyárfás, Z. Tuza, W. T. Trotter, The maximum number of edges in $2 K_{2}$-free graphs of bounded degree, Discrete Math. 81 (1990), no. 2, 129-135.

[5] H. Dao, C. Huneke, J. Schweig, Bounds on the regularity and projective dimension of ideals associated to graphs, J. Algebraic Combin. 38 (2013), no. 1, 37-55.

[6] R. Fröberg, On Stanley-Reisner rings, Topics in algebra, Part 2 (Warsaw, 1988), 57-70, Banach Center Publ., 26, Part 2, PWN, Warsaw, 1990.

[7] J. Herzog, T. Hibi, Monomial Ideals, Springer-Verlag, London, 2011.

[8] J. Herzog, T. Hibi, X. Zheng, Monomial ideals whose powers have a linear resolution, Math. Scand. 95 (2004), no. 1, 23-32.

[9] V. Kodiyalam, Asymptotic behaviour of Castelnuovo-Mumford regularity, Proc. Amer. Math. Soc. 128 (2000), no. 2, 407-411.

[10] M. Moghimian, S. A. Fakhari, S. Yassemi, Regularity of powers of edge ideal of whiskered cycles, Comm. Algebra 45 (2017), no. 3, 1246-1259.

[11] E. Nevo, I. Peeva, $C_{4}$-free edge ideals, J. Algebr. Combin. 37, 243-248 (2013). 